\title{
Lacunes and type 2 diabetes mellitus have a joint effect on cognitive impairment : a retrospective study
}

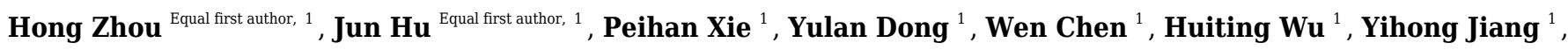 \\ Hao Lei ${ }^{1}$, Guanghua Luo ${ }^{\text {Corresp., } 1}$, Jincai Liu ${ }^{\text {Corresp. } 1}$ \\ ${ }^{1}$ Department of Radiology of the First Affiliated Hospital of University of South China, Unaffiliated, Hengyang, China \\ Corresponding Authors: Guanghua Luo, Jincai Liu \\ Email address: 1579839814@qq.com, liujincai6353@163.com
}

Objective. To evaluate the joint effects of cerebral small vessel disease (CSVD)-related imaging biomarkers in patients of type 2 diabetes mellitus (T2DM) with cognitive impairment.Methods. This study is a retrospective cohort study. A total of 227 participants (115 patients with T2DM and112 healthy control subjects) were enrolled in this study. Cognitive function assessments were evaluated using the Mini-Mental State Examination and the Montreal Cognitive Assessment. The burden of CSVD markers, including the lacunes, white matter hyperintensities (WMH), cerebral microbleeds (CMBs), and enlarged perivascular spaces (PVS), was identified by magnetic resonance imaging and evaluated using small vessel disease (SVD) scores (0-4). The subjects were divided into two groups based on the results of the cognitive function assessments. The synergy index was used to estimate the biological interactions between T2DM and lacunes. Results. There was a significant correlation between T2DM and cognitive impairment ( $p<0.001, \chi^{2}$ test). In patients with diabetes, cognitive impairment was significantly associated with both the presence of lacunes ( $p<0.01, \chi^{2}$ test) and increased total SVD burden scores ( $p$ $<0.01, \chi^{2}$ test). Regarding CMBs, only the existence of lobar CMBs was correlated with cognitive impairment ( $p<0.05, \chi^{2}$ test). The joint effect tended to be larger than the independent effects of T2DM and lacunes on cognitive impairment (adjusted odds ratio [OR]: 7.084, 95\% confidence interval [Cl]: 2.836-17.698; synergy index: $10.018,95 \% \mathrm{Cl}: 0.344-291.414)$.Conclusions. T2DM and the presence of lacunes are significantly correlated with cognitive impairment. There was a joint effect of T2DM and lacunes on cognitive impairment. 


\section{Lacunes and type 2 diabetes mellitus have a joint effect on cognitive}

\section{2 impairment : a retrospective study}

3 Hong Zhou $\dagger^{1}$, Jun $\mathrm{Hu} \dagger^{1}$, Peihan $\mathrm{Xie}^{1}$, Yulan Dong ${ }^{1}$, Wen Chen ${ }^{1}$, Huiting $\mathrm{Wu}^{1}$, Yihong Jiang ${ }^{1}$, 4 Hao Lei ${ }^{1}$, Guanghua Luo*1, Jincai Liu*1

51 Department of Radiology of the First Affiliated Hospital of University of South China, 6 Hengyang, 421001, Hunan, China

$7 \uparrow$ Those authors contributed equally to this work

8 *Corresponding Author:

9 Jincai Liu

10 Department of Radiology of the First Affiliated Hospital of University of South China, Hengyang, 11 421001, China

12 Tel: $+86-13607342347$

13 Fax: +86-0734-8578900

14 E-mail: liujincai6353@163.com

15 Guanghua Luo

16 Department of Radiology of the First Affiliated Hospital of University of South China, Hengyang, 17 421001, China

18 E-mail:1579839814@qq.com

\section{Abstract}

Objective. To evaluate the joint effects of cerebral small vessel disease (CSVD)-related imaging biomarkers in patients of type 2 diabetes mellitus (T2DM) with cognitive impairment.

Methods. This study is a retrospective cohort study. A total of 227 participants (115 patients with T2DM and 112 healthy control subjects) were enrolled in this study. Cognitive function assessments were evaluated using the Mini-Mental State Examination and the Montreal Cognitive Assessment. The burden of CSVD markers, including the lacunes, white matter hyperintensities (WMH), cerebral microbleeds (CMBs), and enlarged perivascular spaces (PVS), was identified by magnetic resonance imaging and evaluated using small vessel disease (SVD) scores (0-4). The subjects were divided into two groups based on the results of the cognitive function assessments. The synergy index was used to estimate the biological interactions between T2DM and lacunes. 
32 Results. There was a significant correlation between T2DM and cognitive impairment $(p<$

$0.001, \chi^{2}$ test). In patients with diabetes, cognitive impairment was significantly associated with both the presence of lacunes ( $p<0.01, \chi^{2}$ test) and increased total SVD burden scores $(p<0.01$, $\chi^{2}$ test). Regarding CMBs, only the existence of lobar CMBs was correlated with cognitive impairment $\left(p<0.05, \chi^{2}\right.$ test). The joint effect tended to be larger than the independent effects of T2DM and lacunes on cognitive impairment (adjusted odds ratio [OR]: 7.084, 95\% confidence interval [CI]: 2.836-17.698; synergy index: 10.018, 95\% CI: 0.344-291.414).

Conclusions. T2DM and the presence of lacunes are significantly correlated with cognitive impairment. There was a joint effect of T2DM and lacunes on cognitive impairment.

Key Words: Cerebral small vessel disease; diabetes; cognitive impairment; magnetic resonance.

\section{Introduction}

Type 2 diabetes mellitus (T2DM) has a very high prevalence worldwide, and the prevalence is increasing year by year. Patients with diabetes often have multiple complications, and cognitive impairment is one of the most common and disruptive complications (Gorelick et al., 2011; Geijselaers et al., 2015; Harreiter \& Roden, 2019b). Cognitive dysfunction not only aggravates deterioration of a patient's physical condition but also causes economic harm (Moon et al., 2021). To date, the relationships between diabetic complications and specific neuropathological causes of cognitive impairment are not fully understood. However, some factors potentially involved in these pathophysiological changes include insulin resistance, high fasting blood glucose, endothelial dysfunction, hypercoagulation, hypertension and comorbid abdominal obesity.

Many epidemiological studies have demonstrated possible associations between diabetes and cerebral small vessel disease (CSVD), in particular with lacunar stroke (Air \& Kissela, 2007; Sorensen et al., 2016; Del Brutto et al., 2018; Liu et al., 2018). The common brain imaging manifestations of diabetes are cerebral infarction and lacunes (Liu et al., 2018; Passiak et al., 2019). Previous studies have shown that diabetes significantly increases the incidence of lacunes. The presence of lacunes has also been associated with reduced cognitive assessment scores (global end-of-life cognition and animal category naming fluency) in patients with diabetes (Abner et al., 2016). It seems that arteriolar perfusion dysfunction can be affected by long-term ischemia and oxidative stress in diabetics (Fernando et al., 2006; Sorensen et al., 2016).Abnormal cerebral blood perfusion can lead to hypoxia in brain tissue through decreased cerebral cortex metabolism and white matter damage, and therefore, diabetic patients often experience cognitive decline (Wallin et al., 2018). The above studies suggest that the existence of CSVD may contribute to cognitive impairment in patients with diabetes.

Magnetic resonance imaging (MRI) is one of the noninvasive imaging modalities for detecting CSVD and it has been standardized to describe various imaging features of CSVD (Wallin et al., 2018). According to international neuroimaging standards, total SVD scores consist of four parts: lacunes, white matter hyperintensities (WMH), cerebral microbleeds (CMBs), and enlarged perivascular spaces (PVS) (Wardlaw et al., 2013). All of these biomarkers 
have been individually linked to some cerebral dysfunction, especially cognitive impairment, but some clinical indicators of CSVD-related markers, such as WMH, CMBs, and lacunes, show poor correlations with neuropsychological performance (Passiak et al., 2019). Accumulated research agrees on the definition of lacunes as a sequela of acute subcortical infarction and that deep cerebral hemorrhages can also form lacunes (Low et al., 2019). Exploring these imaging markers is helpful for studying in diabetes with cognitive impairment.

According to previous studies, the above four neuroimaging biomarkers and their weighted total SVD scores have well-validated associations with vascular risk factors. (Yakushiji et al., 2014; Hara et al., 2019). Therefore, we speculate that the method of quantifying CSVD-related imaging markers can help study the cognitive impairment of patients with diabetes. This study aim to evaluate the joint effects of CSVD-related imaging biomarkers in T2DM with cognitive impairment and investigated whether the SVD score can be used to predict the occurrence of cognitive impairment in diabetic participants.

\section{Materials and Methods}

\subsection{Subjects}

Participants enrolled in the program had to be able to independently complete brain health check tests and undergo MRI scanning for further evaluation. Participants also had to voluntarily accept the regulations provided on the informed consent form. Inclusion criteria: 1. diagnosis criteria for diabetes: Fasting plasma glucose (FPG) $\geq 126 \mathrm{mg} / \mathrm{dl}(7.0 \mathrm{mmol} / \mathrm{l})$, or 2-h values in the oral glucose tolerance test $($ OGTT) $\geq 200 \mathrm{mg} / \mathrm{dl}(11.1 \mathrm{mmol} / \mathrm{l})$, or glycated haemoglobin $(\mathrm{HbA} 1 \mathrm{c}) \geq 6.5 \%$. Exclude other types of diabetes such as type 1 diabetes and gestational diabetes based on clinical diagnosis. (Harreiter \& Roden, 2019a); 2. were over 18 years old and 3. could cooperate and complete the Mini-Mental State Examination, version 2 (MMSE-2) and Montreal Cognitive Assessment (MoCA) conventional tests. The exclusion criteria were as follows: 1. patients had severe brain organic diseases and other serious physical diseases; 2. women were lactating/pregnant; 3. patients had magnetic resonance contraindications; 4. patients had a family history of dementia; 5 . patients refused to sign the informed consent form; or 6. MRI images did not validate the diagnostic criteria.

All 479 participants underwent a 3.0-T MRI scan between May 2017 and January 2019. Individual clinical characteristics, such as age, sex, hyperlipidemia, smoking status, presence of hypertension and diabetes, and years of schooling completed, were collected, and the MMSE-2 and the MoCA were completed. Neuropsychological questionnaires were assessed by a professionally trained nurse in the cognitive function ward. MRI studies were evaluated by two radiologists ( 3 and 9 years of experience in neuroimaging), and controversial MRI images were 
104

105

106

107

108

109

110

111

112

113

114

115

116

117

subsequently evaluated by more experienced radiologists. The two radiologists were blinded to all the clinical information and independently evaluated the characteristics of each patient's brain.

After excluding subjects who had missing or unsatisfactory MRI data for analysis $(\mathrm{n}=50)$, missing basic clinical information $(n=17)$, or missing cognitive assessments $(n=185), 227$ subjects were included in this study (Figure 1). This study was approved by the human ethics review board of the University of South China. All participants provided both oral and written informed consent. The project was registered at the Chinese Clinical Trial Registry (ChiCTR1900027781).

\subsection{Baseline assessment}

According to current diabetes classifications (Harreiter \& Roden, 2019a, 2019b), the definition of T2DM is a fasting serum glucose level $\geq 126 \mathrm{mg} / \mathrm{dl}$ or $\mathrm{HbA} 1 \mathrm{c} \geq 6.5 \%$. Hypertension is defined as systolic blood pressure (SBP) $\geq 140 \mathrm{mmHg}$ and/or diastolic blood pressure $(\mathrm{DBP}) \geq 90 \mathrm{mmHg}$. We systematically collected education information (years of schooling) and smoking history. The MMSE-2 scale defines cognitive impairment as a score $\leq$ 17 points for uneducated participants, $\leq 20$ points for patients with an education period of less than or equal to 6 years, and $\leq 24$ points for patients with an education period of more than 6 years (Cockrell \& Folstein, 1988). Patients with a total score of $<26$ points on the MoCA scale are considered to have cognitive impairment (with an additional point added if the subject has been educated for less than or equal to 12 years) (Nasreddine et al., 2005).

\subsection{Brain MRI acquisition}

Brain MRI scans were obtained at The First Affiliated Hospital of University of South China using the Philips Achieva 3.0-T system with 8-channel SENSE receiver. The main sequences included the following. T1-weighted imaging: repetition time (TR), $600 \mathrm{~ms}$; echo time (TE), 10 ms; flip angle (FA), $70^{\circ}$; section thickness, $5 \mathrm{~mm}$; gap width, $1 \mathrm{~mm}$; matrix, $256 \times 163 \mathrm{~mm}^{2}$; and field of view (FOV), $230 \times 230 \mathrm{~mm}^{2}$. Fast spin-echo T2-weighted imaging: TR, $3000 \mathrm{~ms}$; TE, 80 ms; FA, 90; section thickness, $5 \mathrm{~mm}$; gap width, $1 \mathrm{~mm}$; matrix, $400 \times 255 \mathrm{~mm}^{2}$; and FOV, 230 $\times 230 \mathrm{~mm}^{2}$. Fluid-attenuated inversion recovery (FLAIR) imaging: TR, $11000 \mathrm{~ms}$; inversion time, $2800 \mathrm{~ms}$; TE, $120 \mathrm{~ms}$; FA, 120; section thickness, $4 \mathrm{~mm}$; gap width, $1 \mathrm{~mm}$; matrix, $232 \times$ $101 \mathrm{~mm}^{2}$; and FOV, $230 \times 230 \mathrm{~mm}^{2}$. Susceptibility-weighted imaging (SWI): TR, $18 \mathrm{~ms}$; TE, 26 $\mathrm{ms}$; FA, $10^{\circ}$; section thickness, $0.6 \mathrm{~mm}$; gap width, $1 \mathrm{~mm}$; matrix, $244 \times 200 \mathrm{~mm}^{2}$; and FOV, $220 \times 220 \mathrm{~mm}^{2}$.

\subsection{Lacunes, WMH, CMBs, and PVS analysis}

All evaluators of the MRI examinations were blinded to the clinical data. The MR images were evaluated using the prestudy cerebral SVD study standard (Kim, MacFall \& Payne, 2008; 
138 Gregoire et al., 2009; Wardlaw et al., 2013). Lacunes were defined as focal or ovoid, 3 to $15 \mathrm{~mm}$

139

140

141

142

143

144

145

146

147

148

149

150

151

152

153

154

155

156

157

158

159

160

161

162

163

164

165

166

167

168

169

170

171

172

in diameter, fluid-filled signal cavities located in the area of a perforating arteriole. On FLAIR images, lacunes generally have a central hypointensity, with similar signals as cerebrospinal fluid (CSF), surrounded by hyperintensity (Wardlaw et al., 2013). WMH were defined as hyperintense areas in the T2-weighted image and void-free hyperintense areas on FLAIR sequences. We graded the periventricular and deep lesions according to the modified Fazekas scale.

Periventricular hyperintensities were graded as absent (Grade 0), caps or pencil-thin lining (Grade 1), bands (Grade 2), or irregular extension into the deep white matter (Grade 3), and the deep lesions were graded as absent (Grade 0), punctuate (Grade 1), beginning confluence (Grade 2), or confluent (Grade 3) (Fazekas et al., 1987). We counted the total number of CMBs in the deep, lobar, and infratentorial regions except for lesions in the subarachnoid space and low symmetry signal areas of the globus pallidus (as these possibly represent adjacent pial vessels and calcification, respectively) (Gregoire et al., 2009). The number of CMBs was graded as absent, mild (1-2), moderate (3-10), or severe (>10) (Lee et al., 2002). PVS were defined as small $(<3 \mathrm{~mm})$ structures with the same signal as CSF that followed the area of perforating arteries. The severity of PVS was assessed in the basal ganglia (BG), centrum semiovale, and hippocampus (Hip). In the BG, PVS were graded in the slice that had the largest number of PVS and was scored as follows: the number of PVS $<5$ (Grade 1), 5-10 (Grade 2), $>10$ but still countable (Grade 3), or uncountable (Grade 4). In the centrum semiovale, PVS were scored as follows: the sum of the number of PVS in all slices $<10$ (Grade 1), the sum of the number of PVS in all slices $>10$ but the number of PVS $<10$ in any slice (Grade 2), the number of PVS was 10-20 in the slice containing the largest number of PVS (Grade 3), or the number of PVS > 20 in the slice containing the largest number of PVS (Grade 4) (Doubal et al., 2010). In the Hip, the number of PVS in the total slices of the Hip and parahippocampal gyrus was scored. PVS were scored as follows: number of PVS $<5$ (Grade 1), 5-10 (Grade 2), or $>10$ (Grade 3) (Zhang et al., 2014).

\subsection{SVD score analysis}

In accordance with previous studies (Klarenbeek et al., 2013; Staals et al., 2014), if the number of lacunes or CMBs was greater than or equal to one, they were each recorded as 1 point. The presence of white matter hyperintensities was defined as periventricular WMH Fazekas score 3 and/or deep WMH Fazekas score 3 and recorded as 1 point. The presence of moderate to severe PVS was recorded as 1 point. The total SVD score consists of the above four parts and could range from 0 to 4 . The subjects were further divided based on the total SVD score: a none to mild group (SVD score: 0-1) and a moderate to severe group (SVD score: 2-4) (Yakushiji et al., 2014; Hara et al., 2019).

Peer) reviewing PDF | (2021:08:64569:3:0:NEW 12 Feb 2022) 


\subsection{Statistical analysis}

According to the above baseline assessment criteria, all subjects were divided into two groups (cognitive impairment group and noncognitive impairment group) using the MMSE-2 and MoCA scale. To apply the research to diabetic participants, we selected the appropriate assessment using chi-square or Fisher's exact tests. The subjects were divided into two groups, and all baseline and imaging data were analyzed. Categorical and ordinal variables are presented as $\mathrm{n}(\%)$ and were analyzed with chi-square or Fisher's exact tests. Continuous variables are presented as the mean \pm standard deviation (SD). The normality of data distributions and homogeneity of continuous variables were examined before analysis, and then the appropriate $t$ test was used. The joint effect of diabetes and lacunes was evaluated by calculating the synergy index using the method introduced by Anderson et al (Andersson et al., 2005). An Excel sheet was built to calculate the synergy index. The Excel formula is Synergy index $=\left[R_{11}-1\right] /$ $\left[\left(\mathrm{RR}_{10}-1\right)+\left(\mathrm{RR}_{01}-1\right)\right]$. In this formula, $\mathrm{RR}_{11}$ is the relative risk of both risk factors being present, $\mathrm{RR}_{10}$ is the relative risk of the first risk factor being absent and the second risk factor being present, and $\mathrm{RR}_{01}$ is the relative risk of the second risk factor being absent and the first risk factor being present. If there was no biological interaction, the synergy index is equal to 1 . If the synergy index is larger than 1 , it indicates that there is a synergistic effect of the two factors. Otherwise, there is only a joint effect of the two factors. All statistical analyses were performed with SPSS software (Version 25, SPSS, Chicago, USA), the Statistical Analysis System (Version 9.4, SAS Institute, North Carolina, USA) and Microsoft Excel (Version 2019, Microsoft Corp, WA, USA). Statistical significance was established at $p<0.05$ for all tests.

\section{Results}

\subsection{Participant characteristics}

Cohen's kappa values were calculated as a measure of interrater reliability. The Cohen's kappa values for lacunes, WMH, CMBs, and PVS were 0.76, 0.84, 0.89, and 0.66, respectively. The data were evaluated by two imaging specialists who were unaware of the results of each other's assessment during the assessment period. Table 1 shows the demographic and clinical findings for the entire cohort ( $n=227: 64.3 \%$ male; mean age: 62.5 years). Among the entire cohort, there were 115 (51.7\%) people with diabetes, 74 (32.6\%) people with hyperlipidemia, and $169(74.4 \%)$ people with hypertension. There were $90(39.6 \%)$ people with postsecondary education among the entire cohort and $64(28.2 \%)$ people with a history of smoking.

\subsection{Markers of SVD and cognitive assessment outcomes}

The numbers of subjects with SVD scores of $0,1,2,3$, and 4 were $0(0 \%), 55(24.2 \%), 51$ (22.4\%), 57 (25.8\%), and 64 (28.2\%), respectively. Table 1 shows MMSE-2 scores for the entire 
209

210

211

212

cohort. There was a significant correlation between diabetes and cognitive impairment $(p<$ $0.001, \chi^{2}$ test). The entire cohort was evaluated based on MMSE- 2 scores, and Table 2 shows that total SVD scores were higher in the cognitive impairment group than in the noncognitive impairment group ( $p<0.01, \chi^{2}$ test) and that the existence of lacunes was significantly associated with cognitive impairment ( $p \leq 0.001, \chi^{2}$ test). Table 3 shows the statistics for diabetes patients in the entire cohort that show that increased age ( $p<0.05, \chi^{2}$ test) was associated with cognitive impairment. Table 4 shows no statistically significant differences among the general characteristics in the non-diabetic group. Table 5 shows that in patients with diabetes, cognitive impairment was significantly associated with the presence of lacunes $\left(p<0.01, \chi^{2}\right.$ test) and higher total SVD burden scores $\left(p<0.01, \chi^{2}\right.$ test). Regarding CMBs, only the existence of lobar CMBs was related to cognitive impairment $\left(p<0.05, \chi^{2}\right.$ test).

\subsection{Joint effect of diabetes and lacunes on cognitive impairment}

Table 6 shows that although there were no significant synergistic effects of diabetes and lacunes on cognitive impairment, the joint effects tended to be larger than independent effects after adjusting for sex, age, smoking status, education, hyperlipidemia and hypertension (adjusted OR: 7.084, 95\% CI: 2.836-17.698; synergy index: 10.018, 95\% CI: 0.344-291.414).

\section{Discussion}

In this study, we used MRI to demonstrate changes in small blood vessel damage in diabetic patients with cognitive impairment and to evaluate SVD scores in patients with diabetes with cognitive impairment. Our study showed that the presence of lacunes was significantly connected with cognitive impairment in diabetic patients and that the presence of diabetes and lacunes had a joint effect on the risk of cognitive impairment in addition to increased total SVD burden scores and worse cognitive performance. We have reason to believe that the presence of lacunes is an important imaging biomarker in diabetic patients with cognitive impairment. The presence of lacunes can be used as important evidence for assessing cognitive impairment in patients with diabetes because the appearance of lacunes is significantly linked with the decline in cognitive ability in this population. This study provided further evidence that monitoring lacunes in diabetic patients can help slow the progression of cognitive impairment.

In patients with diabetes, many factors may be involved in these pathophysiological changes, such as insulin resistance, high fasting blood glucose, endothelial dysfunction, and hypercoagulation (Air \& Kissela, 2007; Sorensen et al., 2016; Liu et al., 2018). Arteriolar perfusion dysfunction may be affected by long-term ischemia and oxidative stress (Fernando et al., 2006; Sorensen et al., 2016). Disorders of the blood vessel walls and hyaline accumulation can cause blockage of the arteries, which can lead to the formation of lacunes (Fisher, 1982). For example, an autopsy study of elderly diabetic patients showed that diabetes was significantly associated with lacunes and increased odds of brain lacunes. (Abner et al., 2016). A 4-year 
246 longitudinal study of cognitive function in diabetes showed a moderate cognitive decline

247 (information-processing speed, executive functioning, and memory) in patients with diabetes

248 (van den Berg et al., 2010). Other studies have shown that SVD is a crucial element in diabetic

249 cognitive impairment (Wallin et al., 2018). As one of the factors of small vessel disease, lacunes

250 may mediate the dysfunction of key brain regions. Previous studies have reported that lacunes

251 are linked to decreases in executive function and that there is a significant positive correlation

252 between lacunes and executive function over time (Jokinen et al., 2011; Passiak et al., 2019).

253 The growth of lacunes is mainly located in the subcortical white matter area of the frontal lobe,

254 which may explain the cause of impaired cognitive function, especially executive function

255 (Jokinen et al., 2011). Our study also found that in patients with diabetes, lacune scores were

256 significantly associated with impairments in cognitive function. After further excluding the

257 influence of other factors, we evaluated the correlation between diabetes and lacunes in patients

258 with cognitive impairment and found that the joint effect of lacunes and diabetes on cognitive

259 impairment was stronger than the independent effects of these two factors. We speculate that the

260 simultaneous existence of these two factors is also correlated with greater damage to cognitive

261 function. At present, there is a lack of sufficient research to investigate the cause of this

262 phenomenon, and we intend to study this topic further.

263 Lobar CMBs might also play a key role in cognitive impairment (Yakushiji \& Werring, 2016).

264 In this study, we also confirmed that a high score for lobar CMBs was correlated with

265 significantly increased cognitive impairment in patients with diabetes, which may help explain

266 the deficits in global cognitive and visuospatial executive functions observed in diabetic patients

267 (Yakushiji \& Werring, 2016). Our study showed no significant differences in WMH scores and

268 PVS scores between the cognitive impairment and noncognitive impairment groups, which

269 differs from previous research (Passiak et al., 2019). All subjects in the study cohort had a PVS

270 score of 1 . This situation is because even healthy people monitored the presence of PVS in this

271 study. A study about PVS in neurologically healthy people also confirmed this phenomenon

272 (Yakushiji et al., 2014). Therefore, the feasibility of evaluating cognitive impairment based on

273 PVS score data alone needs to be further explored. Although studies have shown that PVS is

274 associated with worse cognitive function (Passiak et al., 2019), this study did not find that grade

275 of PVS is statistically significant with cognitive impairment in diabetic patients. This situation

276 may be due to the older patients in this study. Studies have shown that age, hypertension, and

277 genetic factors are all related to PVS (Wardlaw et al., 2013; Duperron et al., 2018; Passiak et al.,

278 2019). Because research shows that age, hypertension, and genetic factors are all related to PVS,

279 advanced age may obscure meaningful information. Expanding the sample size may be able to

280 solve this problem. The weakened association in the clinical radiological assessment of SVD

281 caused by the heterogeneity in the pathological substrates of WMH (Gouw et al., 2011) may

282 explain why WMH had no significant statistical associations with diabetic cognitive impairment

283 in this study.

284 This study still has some limitations. First, age is one of the important factors affecting

285 cognitive function. This study found that the presence of diabetes and lacunes had a joint effect

286 on the risk of cognitive impairment, but this may be because the overall sample in this study was 
287

288

289

290

291

292

293

294

older. A sample with a larger age range would be required to further understand the interaction of these two factors on cognitive impairment (such as whether there are synergistic effects in particular age groups) and to strengthen our current research conclusions. Second, due to technological limitations, we did not further study the effects of more than two risk factor interactions on cognitive function. Third, we did not have pathologic confirmation of the type of small vessel disease (SVD) in our participants. There was some information about SVD in the participants, but some vascular risk factors, including atrial fibrillation and carotid plaque, were lacking. Fourth, possible geographical biases may have resulted in a low level of education in this group of subjects. Similar to recent research (Passiak et al., 2019), educational background was also one of the factors associated with cardiovascular disease. Further investigation may be needed to clarify whether our findings are more generally applicable. Finally, because of the cross-sectional nature of this study, we were unable to determine the specific time of cognitive dysfunction and the progression of cognitive impairment in these patients with diabetes. Tracking the detected neuroimaging biomarkers in patients with diabetes with mild cognitive impairment might help solve this problem.

\section{Conclusion}

The assessment of small vessel disease in diabetic patients with cognitive impairment found that the existence of lacunes was significantly related to cognitive dysfunction. In addition, diabetes and lacunes have a joint effect on cognitive impairment. These results suggest that monitoring lacunes, as a neuroimaging biomarker, may help assess the deterioration in cognitive function in diabetic patients.

\section{Conflict of Interest}

The authors declare that they have no actual or potential conflicts of interest.

\section{Acknowledgments}

Hong Zhou, Jun Hu contributed equally to this work and analyzed literature and organized the document together. Jincai Liu and Guanghua Luo: Conceptualization, Project administration, Funding acquisition, Conceptualization Supervision. Peihan Xie: Visualization, Data Curation. Yulan Dong: Resources, Conceptualization. Wen Chen: Formal analysis, Investigation. Huiting Wu: Investigation. Yihong Jiang: Investigation, Project administration. Hao Lei: Methodology, Data curation. 


\section{References}

Abner EL, Nelson PT, Kryscio RJ, Schmitt FA, Fardo DW, Woltjer RL, Cairns NJ, Yu L, Dodge HH, Xiong C, Masaki K, Tyas SL, Bennett DA, Schneider JA, Arvanitakis Z. 2016. Diabetes is associated with cerebrovascular but not Alzheimer's disease neuropathology. Alzheimer's \& dementia : the journal of the Alzheimer's Association 12 (8):882-889.

Air EL, Kissela BM. 2007. Diabetes, the metabolic syndrome, and ischemic stroke: epidemiology and possible mechanisms. Diabetes Care 30 (12):3131-3140.

Andersson T, Alfredsson L, Källberg H, Zdravkovic S, Ahlbom A. 2005. Calculating measures of biological interaction. European journal of epidemiology 20 (7):575-579.

Cockrell JR, Folstein MF. 1988. Mini-Mental State Examination (MMSE). Psychopharmacology bulletin 24 (4):689-692.

Del Brutto VJ, Ortiz JG, Del Brutto OH, Mera RM, Zambrano M, Biller J. 2018. Total cerebral small vessel disease score and cognitive performance in community-dwelling older adults. Results from the Atahualpa Project. International journal of geriatric psychiatry 33 (2):325-331.

Doubal FN, MacLullich AMJ, Ferguson KJ, Dennis MS, Wardlaw JM. 2010. Enlarged perivascular spaces on MRI are a feature of cerebral small vessel disease. Stroke 41 (3):450-454.

Duperron M-G, Tzourio C, Sargurupremraj M, Mazoyer B, Soumaré A, Schilling S, Amouyel P, Chauhan G, Zhu Y-C, Debette S. 2018. Burden of Dilated Perivascular Spaces, an Emerging Marker of Cerebral Small Vessel Disease, Is Highly Heritable. Stroke 49 (2):282-287.

Fazekas F, Chawluk JB, Alavi A, Hurtig HI, Zimmerman RA. 1987. MR signal abnormalities at $1.5 \mathrm{~T}$ in Alzheimer's dementia and normal aging. AJR. American journal of roentgenology 149 (2):351-356.

Fernando MS, Simpson JE, Matthews F, Brayne C, Lewis CE, Barber R, Kalaria RN, Forster G, Esteves F, Wharton SB, Shaw PJ, O'Brien JT, Ince PG. 2006. White matter lesions in an unselected cohort of the elderly: molecular pathology suggests origin from chronic hypoperfusion injury. Stroke 37 (6):1391-1398.

Fisher CM. 1982. Lacunar strokes and infarcts: a review. Neurology 32 (8):871-876.

Geijselaers SLC, Sep SJS, Stehouwer CDA, Biessels GJ. 2015. Glucose regulation, cognition, and brain MRI in type 2 diabetes: a systematic review. The Lancet Diabetes \& Endocrinology 3 (1):75-89.

Gorelick PB, Scuteri A, Black SE, Decarli C, Greenberg SM, Iadecola C, Launer LJ, Laurent S, Lopez OL, Nyenhuis D, Petersen RC, Schneider JA, Tzourio C, Arnett DK, Bennett DA, Chui HC, Higashida RT, Lindquist R, Nilsson PM, Roman GC, Sellke FW, Seshadri S. 2011. Vascular contributions to cognitive impairment and dementia: a statement for healthcare professionals from the american heart association/american stroke association. Stroke 42 (9):2672-2713.

Gouw AA, Seewann A, van der Flier WM, Barkhof F, Rozemuller AM, Scheltens P, Geurts JJG. 2011. Heterogeneity of small vessel disease: a systematic review of MRI and histopathology correlations. Journal of neurology, neurosurgery, and psychiatry 82 (2):126-135.

Gregoire SM, Chaudhary UJ, Brown MM, Yousry TA, Kallis C, Jäger HR, Werring DJ. 2009. The 
Microbleed Anatomical Rating Scale (MARS): reliability of a tool to map brain microbleeds. Neurology 73 (21): 1759-1766.

Hara M, Yakushiji Y, Suzuyama K, Nishihara M, Eriguchi M, Noguchi T, Nishiyama M, Nanri Y, Tanaka J, Hara H. 2019. Synergistic effect of hypertension and smoking on the total small vessel disease score in healthy individuals: the Kashima scan study. Hypertension research : official journal of the Japanese Society of Hypertension.

Harreiter J, Roden M. 2019a. Diabetes mellitus - Definition, Klassifikation, Diagnose, Screening und Prävention (Update 2019). Wiener klinische Wochenschrift 131 (Suppl 1):6-15.

Harreiter J, Roden M. 2019b. Diabetes mellitus - Definition, Klassifikation, Diagnose, Screening und Prävention (Update 2019). Wiener klinische Wochenschrift 131 (Suppl 1):6-15.

Jokinen H, Gouw AA, Madureira S, Ylikoski R, van Straaten ECW, van der Flier WM, Barkhof F, Scheltens P, Fazekas F, Schmidt R, Verdelho A, Ferro JM, Pantoni L, Inzitari D, Erkinjuntti T. 2011. Incident lacunes influence cognitive decline: the LADIS study. Neurology 76 (22):1872-1878.

Kim KW, MacFall JR, Payne ME. 2008. Classification of white matter lesions on magnetic resonance imaging in elderly persons. Biological psychiatry 64 (4):273-280.

Klarenbeek P, van Oostenbrugge RJ, Lodder J, Rouhl RPW, Knottnerus ILH, Staals J. 2013. Higher ambulatory blood pressure relates to enlarged Virchow-Robin spaces in first-ever lacunar stroke patients. Journal of neurology 260 (1):115-121.

Lee S-H, Bae H-J, Yoon B-W, Kim H, Kim D-E, Roh J-K. 2002. Low concentration of serum total cholesterol is associated with multifocal signal loss lesions on gradient-echo magnetic resonance imaging: analysis of risk factors for multifocal signal loss lesions. Stroke 33 (12):2845-2849.

Liu J, Rutten-Jacobs L, Liu M, Markus HS, Traylor M. 2018. Causal Impact of Type 2 Diabetes Mellitus on Cerebral Small Vessel Disease: A Mendelian Randomization Analysis. Stroke 49 (6):1325-1331.

Low A, Mak E, Rowe JB, Markus HS, O'Brien JT. 2019. Inflammation and cerebral small vessel disease: A systematic review. Ageing research reviews 53:100916.

Moon W, Han JW, Bae JB, Suh SW, Kim TH, Kwak KP, Kim BJ, Kim SG, Kim JL, Moon SW, Park JH, Ryu S-H, Youn JC, Lee DY, Lee DW, Lee SB, Lee JJ, Jhoo JH, Kim KW. 2021. Disease Burdens of Alzheimer's Disease, Vascular Dementia, and Mild Cognitive Impairment. Journal of the American Medical Directors Association 22 (10):2093-2099.e3.

Nasreddine ZS, Phillips NA, Bédirian V, Charbonneau S, Whitehead V, Collin I, Cummings JL, Chertkow H. 2005. The Montreal Cognitive Assessment, MoCA: a brief screening tool for mild cognitive impairment. Journal of the American Geriatrics Society 53 (4):695-699.

Passiak BS, Liu D, Kresge HA, Cambronero FE, Pechman KR, Osborn KE, Gifford KA, Hohman TJ, Schrag MS, Davis LT, Jefferson AL. 2019. Perivascular spaces contribute to cognition beyond other small vessel disease markers. Neurology 92 (12):e1309-e1321.

Sorensen BM, Houben AJ, Berendschot TT, Schouten JS, Kroon AA, van der Kallen, C. J., Henry RM, Koster A, Sep SJ, Dagnelie PC, Schaper NC, Schram MT, Stehouwer CD. 2016. Prediabetes and Type 2 Diabetes Are Associated With Generalized Microvascular Dysfunction: The Maastricht Study. Circulation 134 (18):1339-1352.

Staals J, Makin SDJ, Doubal FN, Dennis MS, Wardlaw JM. 2014. Stroke subtype, vascular risk factors, and total MRI brain small-vessel disease burden. Neurology 83 (14):1228-1234. 
van den Berg E, Reijmer YD, Bresser J de, Kessels RPC, Kappelle LJ, Biessels GJ. 2010. A 4 year followup study of cognitive functioning in patients with type 2 diabetes mellitus. Diabetologia 53 (1):58-65.

Wallin A, Roman GC, Esiri M, Kettunen P, Svensson J, Paraskevas GP, Kapaki E. 2018. Update on Vascular Cognitive Impairment Associated with Subcortical Small-Vessel Disease. J Alzheimers Dis 62 (3):1417-1441.

Wardlaw JM, Smith EE, Biessels GJ, Cordonnier C, Fazekas F, Frayne R, Lindley RI, O'Brien JT, Barkhof F, Benavente OR, Black SE, Brayne C, Breteler M, Chabriat H, DeCarli C, Leeuw F-E de, Doubal F, Duering M, Fox NC, Greenberg S, Hachinski V, Kilimann I, Mok V, van Oostenbrugge R, Pantoni L, Speck O, Stephan BCM, Teipel S, Viswanathan A, Werring D, Chen C, Smith C, van Buchem M, Norrving B, Gorelick PB, Dichgans M. 2013. Neuroimaging standards for research into small vessel disease and its contribution to ageing and neurodegeneration. The Lancet Neurology 12 (8):822-838.

Yakushiji Y, Charidimou A, Hara M, Noguchi T, Nishihara M, Eriguchi M, Nanri Y, Nishiyama M, Werring DJ, Hara H. 2014. Topography and associations of perivascular spaces in healthy adults: the Kashima scan study. Neurology 83 (23):2116-2123.

Yakushiji Y, Werring DJ. 2016. Cerebrovascular disease: Lobar cerebral microbleeds signal early cognitive impairment. Nature reviews. Neurology 12 (12):680-682.

Zhang C, Chen Q, Wang Y, Zhao X, Wang C, Liu L, Pu Y, Zou X, Du W, Pan Y, Li Z, Jing J, Wang D, Luo Y, Wong KS, Wang Y. 2014. Risk factors of dilated Virchow-Robin spaces are different in various brain regions. PloS one $\mathbf{9}$ (8):e105505. 
Figure 1

the whole study flowchart (Recruitment process of study participants) 


\begin{tabular}{|c|c|}
\hline \multicolumn{2}{|c|}{ Participants with TIA or $\mathrm{Cl} \quad(n=479)$} \\
\hline & MRI missing and can not evaluate $(n=50)$ \\
\hline \multicolumn{2}{|c|}{ Participants with MRI （ $n=429 ）$} \\
\hline & Basic clinical information missing $(n=17)$ \\
\hline \multicolumn{2}{|c|}{ Participants with sufficient information $(n=412)$} \\
\hline$\downarrow$ & Cognitive assessment missing $\quad(n=185)$ \\
\hline \multicolumn{2}{|c|}{ Participants with MRI and Cognitive assessment $(n=227)$} \\
\hline \multicolumn{2}{|c|}{$\downarrow$} \\
\hline \multicolumn{2}{|c|}{ Divide into groups by neuropsychological scale $(n=227)$} \\
\hline$\downarrow$ & $\downarrow$ \\
\hline Participants without cognitive impairment & Participants with cognitive impairment \\
\hline
\end{tabular}

MRI: Magnetic Resonance Imaging. 


\section{Table $\mathbf{1}_{\text {(on next page) }}$}

\section{General characteristics of the study population}

PSE: post-secondary education. MMSE: Mini-mental State Examination. MOCA: Montreal Cognitive Assessment. Cl: cognitive impairment.

A statistical analysis of age found that when using the MMSE-2 assessment, the non-cognitive group had a normal distribution and the cognitively disabled group did not follow the normal distribution. Therefore, a non-parametric test was selected. When using the MMSE-2 assessment, the non-cognitive disorder group had a normal distribution, and the cognitively impaired group did not follow the normal distribution. Therefore, a non-parametric test was selected. Chi-square tests were performed on sex, education, diabetes, smoking, hypertension, and hyperlipidemia. Among the MOCA assessment groups, the number of individual tests for diabetes and gender was less than 5 , so the continuous correction value test was used, and the others were routinely selected by Pearson's chi-squared test. There were statistical differences in the MMSE-2 assessment in the diabetic group, so the MMSE-2 was selected as a scale for cognitive assessment before subsequent analysis. 


\section{General characteristics of the study population}

\begin{tabular}{|c|c|c|c|c|c|c|c|}
\hline & $\begin{array}{l}\text { Entire cohort } \\
\qquad(\mathrm{n}=227)\end{array}$ & \multicolumn{3}{|c|}{ Evaluated by using MMSE-2 } & \multicolumn{3}{|c|}{ Evaluated by using MOCA } \\
\hline Age (median) & $62.59 \pm 10.84$ & $61.65 \pm 11.00$ & $64.64 \pm 10.27$ & 0.053 & $59.06 \pm 12.12$ & $62.86 \pm 10.72$ & 0.211 \\
\hline Education (PSE, \%) & $90(39.6 \%)$ & $58(37.4 \%)$ & $32(44.4 \%)$ & 0.314 & $10(62.5 \%)$ & $80(37.9 \%)$ & 0.053 \\
\hline Smoke & $64(28.2 \%)$ & $49(31.6 \%)$ & $15(20.8 \%)$ & 0.093 & $6(37.5 \%)$ & $58(27.5 \%)$ & 0.396 \\
\hline Hyperlipidemia & $74(32.6 \%)$ & $46(29.7 \%)$ & $28(38.9 \%)$ & 0.168 & $5(31.3 \%)$ & $69(32.7 \%)$ & 0.905 \\
\hline Hypertension (\%) & $169(74.4 \%)$ & $109(70.3 \%)$ & $60(83.3 \%)$ & 0.036 & $8(50.0 \%)$ & $161(76.3 \%)$ & 0.108 \\
\hline
\end{tabular}

A statistical analysis of age found that when using the MMSE-2 assessment, the non-cognitive group had a normal distribution and the cognitively disabled group did not follow the normal distribution. Therefore, a non-parametric test was selected. When using the MMSE-2 assessment, the non-cognitive disorder group had a normal distribution, and the cognitively impaired group did not follow the normal distribution. Therefore, a non-parametric test was selected. Chisquare tests were performed on sex, education, diabetes, smoking, hypertension, and hyperlipidemia. Among the MOCA assessment groups, the number of individual tests for diabetes and gender was less than 5, so the continuous correction value test was used, and the others were routinely selected by Pearson's chisquared test. There were statistical differences in the MMSE-2 assessment in the diabetic group, so the MMSE-2 was selected as a scale for cognitive assessment

before subsequent analysis. 


\section{Table 2 (on next page)}

Small blood vessel scores using MMSE-2 scoring in the entire cohort.

WMH: white matter hyperintensities. CMBs: cerebral microbleeds. PVS: perivascular spaces. SVD: small vessel disease. MMSE: Mini-mental State Examination.Cl: cognitive impairment.

Statistical tests showed that the data all conformed to the normal distribution with or without cognitive impairment, and then the homogeneity of variance was further tested. ANOVA analysis was used to conclude that the total CMBs score, total lacunes score, lobar CMBs score, total SVD burden score and total SVD burden score $\geq 3$ was heterogeneity of variance, therefore, Welch's adjusted unpaired $t$-test was selected. For others, an unpaired $t$-test was selected. 
2 Small blood vessel scores using MMSE-2 scoring in the entire cohort

\begin{tabular}{|c|c|c|c|c|c|}
\hline & \multicolumn{5}{|c|}{ Evaluated by using MMSE-2 } \\
\hline & Without CI & With CI & $\mathrm{F}$ & $t$ & $p$ \\
\hline Total WMH score & $0.39 \pm 0.490$ & $0.51 \pm 0.503$ & 3.247 & -1.707 & 0.089 \\
\hline Periventricular WMH & $1.43 \pm 0.925$ & $1.64 \pm 0.954$ & 3.247 & -1.599 & 0.111 \\
\hline Deep WMH & $0.39 \pm 0.490$ & $0.51 \pm 0.503$ & 0.497 & -1.707 & 0.093 \\
\hline Total CMBs score & $0.60 \pm 0.491$ & $0.71 \pm 0.458$ & 12.128 & -1.579 & 0.116 \\
\hline Lobar CMBs & $0.31 \pm 0.464$ & $0.43 \pm 0.499$ & 8.485 & -1.737 & 0.085 \\
\hline Deep CMBs & $0.48 \pm 0.501$ & $0.60 \pm 0.494$ & 4.822 & -1.693 & 0.094 \\
\hline subtentorial CMBs & $0.26 \pm 0.439$ & $0.26 \pm 0.444$ & 0.034 & -0.093 & 0.926 \\
\hline Total Lacunes score & $0.45 \pm 0.499$ & $0.68 \pm 0.470$ & 15.489 & -3.446 & $\leqslant 0.001$ \\
\hline Total perivascular spaces score & $1.00 \pm 0.000$ & $1.00 \pm 0.000$ & & & \\
\hline Basal ganglia PVS & $3.11 \pm 0.778$ & $3.22 \pm 0.859$ & 3.065 & -0.981 & 0.328 \\
\hline Centrum semiovale PVS & $3.46 \pm 0.647$ & $3.54 \pm 0.670$ & 0.030 & -0.896 & 0.371 \\
\hline Hippocampus PVS & $2.32 \pm 0.728$ & $2.50 \pm 0.732$ & 0.041 & -1.769 & 0.078 \\
\hline Total SVD burden score & $2.42 \pm 1.167$ & $2.90 \pm 1.009$ & 10.248 & -3.192 & $<0.01$ \\
\hline$\geq 2$ & $3.02 \pm 0.850$ & $3.17 \pm 0.752$ & 1.805 & -1.211 & 0.228 \\
\hline$\geq 3$ & $3.56 \pm 0.499$ & $3.48 \pm 0.505$ & 0.608 & 0.900 & 0.370 \\
\hline
\end{tabular}

WMH: white matter hyperintensities. CMBs: cerebral microbleeds. PVS: perivascular spaces. SVD: small vessel disease. MMSE: Mini-mental State

Examination. CI: cognitive impairment.

Statistical tests showed that the data all conformed to the normal distribution with or without cognitive impairment, and then the homogeneity of variance was

6 further tested. ANOVA analysis was used to conclude that the total CMBs score, total lacunes score, lobar CMBs score, total SVD burden score and total SVD

7 burden score $\geq 3$ was heterogeneity of variance, therefore, Welch's adjusted unpaired $t$-test was selected. For others, an unpaired $t$-test was selected. 


\section{Table 3(on next page)}

General characteristics of diabetic patients in this cohort

PSE: post-secondary education. MMSE: Mini-mental State Examination. Cl: cognitive impairment. DM: diabetes mellitus

Statistical analysis of age revealed that both groups conformed to a normal distribution and the variance tests were not homogeneous. Therefore, Welch's adjusted unpaired $t$-test was selected. For other factors, a chi-square test was selected. 
2 General characteristics of diabetic patients in this cohort

\begin{tabular}{|c|c|c|c|c|c|}
\hline & \multirow{2}{*}{$\begin{array}{l}\text { Entire cohort } \\
\qquad(\mathrm{n}=115)\end{array}$} & \multicolumn{4}{|c|}{ Evaluated by using MMSE-2 } \\
\hline & & $\begin{array}{l}\text { DM Without CI } \\
\quad(\mathrm{n}=60)\end{array}$ & $\begin{array}{l}\text { DM With CI } \\
(\mathrm{n}=55)\end{array}$ & $\chi^{2 / t}$ & $p$ \\
\hline Sex (men, \%) & $65(56.5 \%)$ & $35(58.3 \%)$ & $30(54.5 \%)$ & 0.168 & 0.682 \\
\hline Age (median) & $62.42 \pm 9.382$ & $62.52 \pm 9.038$ & $66.49 \pm 9.390$ & -2.312 & $<0.05$ \\
\hline Education (PSE, \%) & $39(33.9 \%)$ & $17(28.3 \%)$ & $22(40.0 \%)$ & 1.743 & 0.187 \\
\hline Smoke & $29(25.2 \%)$ & $17(28.3 \%)$ & $12(21.8 \%)$ & 0.646 & 0.422 \\
\hline Hyperlipidemia & $36(31.3 \%)$ & $16(26.7 \%)$ & $20(36.4 \%)$ & 1.255 & 0.263 \\
\hline Hypertension (\%) & $96(83.5 \%)$ & $49(81.7 \%)$ & $47(85.5 \%)$ & 0.299 & 0.585 \\
\hline
\end{tabular}

PSE: post-secondary education. MMSE: Mini-mental State Examination. CI: cognitive impairment. DM: diabetes mellitus

4 Statistical analysis of age revealed that both groups conformed to a normal distribution and the variance tests were not homogeneous. Therefore, Welch's adjusted 5 unpaired $t$-test was selected. For other factors, a chi-square test was selected. 


\section{Table 4(on next page)}

\section{General characteristics of non-diabetic patients in this cohort}

PSE: post-secondary education. MMSE: Mini-mental State Examination. Cl: cognitive impairment.

Statistical analysis of age revealed that both groups conformed to a normal distribution and the variance tests were not homogeneous. Therefore, Welch's adjusted unpaired $t$-test was selected. For other factors, a chi-square test was selected. 
2 General characteristics of non-diabetic patients in this cohort

\begin{tabular}{|c|c|c|c|c|c|}
\hline & \multirow{2}{*}{$\begin{array}{l}\text { Entire cohort } \\
\qquad(\mathrm{n}=112)\end{array}$} & \multicolumn{4}{|c|}{ Evaluated by using MMSE-2 } \\
\hline & & $\begin{array}{l}\text { Without CI } \\
(\mathrm{n}=95)\end{array}$ & $\begin{array}{l}\text { With CI } \\
(\mathrm{n}=17)\end{array}$ & $\chi^{2} / t$ & $p$ \\
\hline Sex (men, \%) & $81(72.3 \%)$ & $72(75.8 \%)$ & $9(52.9 \%)$ & 3.761 & 0.076 \\
\hline Age (median) & $60.72 \pm 11.907$ & $61.09 \pm 12.083$ & $58.65 \pm 10.977$ & 0.779 & 0.438 \\
\hline Education (PSE, \%) & $51(45.5 \%)$ & $41(43.2 \%)$ & $10(58.8 \%)$ & 1.427 & 0.232 \\
\hline Smoke & $35(31.3 \%)$ & $32(33.7 \%)$ & $3(17.6 \%)$ & 1.726 & 0.189 \\
\hline Hyperlipidemia & $38(33.9 \%)$ & $30(31.6 \%)$ & $8(47.1 \%)$ & 1.541 & 0.214 \\
\hline Hypertension (\%) & $73(65.2 \%)$ & $60(63.2 \%)$ & $13(81.5 \%)$ & 1.126 & 0.289 \\
\hline
\end{tabular}

PSE: post-secondary education. MMSE: Mini-mental State Examination. CI: cognitive impairment.

4 Statistical analysis of age revealed that both groups conformed to a normal distribution and the variance tests were not homogeneous. Therefore, Welch's

5 adjusted unpaired $t$-test was selected. For other factors, a chi-square test was selected. 


\section{Table 5 (on next page)}

Small blood vessel scores using MMSE-2 scoring in diabetic

WMH: white matter hyperintensities. CMBs: cerebral microbleeds. PVS: perivascular spaces. SVD: small vessel disease. MMSE: Mini-mental State Examination. $\mathrm{Cl}$ : cognitive impairment.

All data were statistically tested for normal distribution. ANOVA analysis was used to test the homogeneity of variance and found that the total Lacunes score, lobar CMBs score, total SVD burden score and total SVD burden score $\geq 3$ was heterogeneity of variance. Therefore, Welch's adjusted unpaired $t$-test was selected. For others, an unpaired $t$-test was selected. 
2 Small blood vessel scores using MMSE-2 scoring in diabetic

\begin{tabular}{|c|c|c|c|c|c|}
\hline & \multicolumn{5}{|c|}{ Evaluated by using MMSE-2 } \\
\hline & Without CI & With CI & $\mathrm{F}$ & $t$ & $p$ \\
\hline Total WMH score & $0.50 \pm 0.504$ & $0.58 \pm 0.498$ & 1.622 & -0.875 & 0.384 \\
\hline Periventricular WMH & $1.58 \pm 0.962$ & $1.76 \pm 0.922$ & 0.285 & -1.024 & 0.308 \\
\hline Deep WMH & $0.50 \pm 0.504$ & $0.58 \pm 0.498$ & 1.622 & -0.875 & 0.384 \\
\hline Total CMBs score & $0.73 \pm 0.446$ & $0.82 \pm 0.389$ & 4.840 & -1.089 & 0.278 \\
\hline Lobar CMBs & $0.30 \pm 0.462$ & $0.51 \pm 0.505$ & 5.340 & -2.311 & $<0.05$ \\
\hline Deep CMBs & $0.63 \pm 0.486$ & $0.69 \pm 0.466$ & 1.672 & -0.647 & 0.519 \\
\hline subtentorial CMBs & $0.27 \pm 0.446$ & $0.25 \pm 0.440$ & 0.086 & 0.147 & 0.884 \\
\hline Total Lacunes score & $0.52 \pm 0.504$ & $0.78 \pm 0.417$ & 27.107 & -3.059 & $<0.01$ \\
\hline Total perivascular spaces score & $1.00 \pm 0.000$ & $1.00 \pm 0.000$ & & & \\
\hline Basal ganglia PVS & $3.18 \pm 0.676$ & $3.33 \pm 0.771$ & 4.130 & -1.060 & 0.291 \\
\hline Centrum semiovale PVS & $3.52 \pm 0.624$ & $3.62 \pm 0.552$ & 3.109 & -1.251 & 0.214 \\
\hline Hippocampus PVS & $2.40 \pm 0.669$ & $2.62 \pm 0.593$ & 2.605 & -1.844 & 0.068 \\
\hline Total SVD burden score & $2.68 \pm 1.097$ & $3.18 \pm 0.884$ & 7.508 & -2.693 & $<0.01$ \\
\hline$\geq 2$ & $3.02 \pm 0.869$ & $3.31 \pm 0.729$ & 1.890 & -1.815 & 0.073 \\
\hline$\geq 3$ & $3.59 \pm 0.499$ & $3.55 \pm 0.504$ & 0.686 & 0.414 & 0.680 \\
\hline
\end{tabular}

WMH: white matter hyperintensities. CMBs: cerebral microbleeds. PVS: perivascular spaces. SVD: small vessel disease. MMSE: Mini-mental State

Examination. CI: cognitive impairment.

5 All data were statistically tested for normal distribution. ANOVA analysis was used to test the homogeneity of variance and found that the total Lacunes score,

6 lobar CMBs score, total SVD burden score and total SVD burden score $\geq 3$ was heterogeneity of variance. Therefore, Welch's adjusted unpaired $t$-test was

7 selected. For others, an unpaired $t$-test was selected. 


\section{Table 6(on next page)}

Joint effect of diabetes and lacunas to cognitive impairment.

Abbreviations: $\mathrm{Cl}$, confidence interval; OR, odds ratio; ref, reference.

${ }^{a}$ without any adjusted.

${ }^{\mathrm{b}}$ adjusted for age.

${ }^{c}$ adjusted for sex and age.

d adjusted for sex, age, and history of smoking.

${ }^{\mathrm{e}}$ adjusted for age, sex, history of smoking, and education.

${ }^{f}$ adjusted for sex, age, smoking status, education, and hyperlipidemia.

${ }^{g}$ adjusted for sex, age, smoking status, education, hyperlipidemia, and hypertension. 
1 Table 6

2 Joint effect of diabetes and lacunas to cognitive impairment

\begin{tabular}{|c|c|c|c|c|c|}
\hline \multirow[t]{2}{*}{ Factor1 } & \multirow[t]{2}{*}{ Factor2 } & \multirow{2}{*}{\multicolumn{2}{|c|}{$\begin{array}{c}\begin{array}{c}\text { Without Cognitive } \\
\text { Impairment }\end{array} \\
\mathrm{N}\end{array}$}} & \multicolumn{2}{|c|}{ Cognitive Impairment } \\
\hline & & & & Adjusted OR & $(95 \% \mathrm{CI})$ \\
\hline diabetes & lacuna & & & & \\
\hline$(-)$ & $(-)$ & 57 & 11 & 1.00 & (ref.) \\
\hline$(-)$ & $(+)$ & 38 & 6 & $0.818^{\mathrm{a}}$ & $(0.279-2.400)$ \\
\hline$(+)$ & $(-)$ & 29 & 12 & $2.144^{\mathrm{a}}$ & $(0.844-5.477)$ \\
\hline \multirow[t]{2}{*}{$(+)$} & $(+)$ & 31 & 43 & $7.188^{\mathrm{a}}$ & $(3.250-15.895)$ \\
\hline & & & Synergy index & $6.429^{a}$ & $(0.753-54.911)$ \\
\hline diabetes & lacuna & & & & \\
\hline$(-)$ & $(-)$ & 57 & 11 & 1.00 & (ref.) \\
\hline$(-)$ & $(+)$ & 38 & 6 & $0.674^{\mathrm{b}}$ & $(0.218-2.081)$ \\
\hline$(+)$ & $(-)$ & 29 & 12 & $1.905^{\mathrm{b}}$ & $(0.734-4.944)$ \\
\hline \multirow[t]{2}{*}{$(+)$} & $(+)$ & 31 & 43 & $6.248^{\mathrm{b}}$ & $(2.743-14.230)$ \\
\hline & & & Synergy index & $9.059^{b}$ & $(0.316-259.394)$ \\
\hline diabetes & lacuna & & & & \\
\hline$(-)$ & $(-)$ & 57 & 11 & 1.00 & (ref.) \\
\hline$(-)$ & $(+)$ & 38 & 6 & $0.730^{\mathrm{c}}$ & $(0.234-2.273)$ \\
\hline$(+)$ & $(-)$ & 29 & 12 & $1.779^{\mathrm{c}}$ & $(0.679-4.663)$ \\
\hline \multirow[t]{2}{*}{$(+)$} & $(+)$ & 31 & 43 & $6.221^{\mathrm{c}}$ & $(2.719-14.235)$ \\
\hline & & & Synergy index & $10.256^{c}$ & $(0.236-445.414)$ \\
\hline diabetes & lacuna & & & & \\
\hline$(-)$ & $(-)$ & 57 & 11 & 1.00 & (ref.) \\
\hline$(-)$ & $(+)$ & 38 & 6 & $0.754^{\mathrm{d}}$ & $(0.242-2.353)$ \\
\hline$(+)$ & $(-)$ & 29 & 12 & $1.807^{\mathrm{d}}$ & $(0.688-4.746)$ \\
\hline \multirow[t]{2}{*}{$(+)$} & $(+)$ & 31 & 43 & $6.411^{\mathrm{d}}$ & $(2.785-14.755)$ \\
\hline & & & Synergy index & $9.647^{d}$ & $(0.302-308.531)$ \\
\hline diabetes & lacuna & & & & \\
\hline$(-)$ & $(-)$ & 57 & 11 & 1.00 & (ref.) \\
\hline$(-)$ & $(+)$ & 38 & 6 & $0.705^{\mathrm{e}}$ & $(0.222-2.241)$ \\
\hline$(+)$ & $(-)$ & 29 & 12 & $1.959^{\mathrm{e}}$ & $(0.735-5.221)$ \\
\hline \multirow[t]{2}{*}{$(+)$} & $(+)$ & 31 & 43 & $7.179^{\mathrm{e}}$ & $(3.038-16.965)$ \\
\hline & & & Synergy index & $9.301^{\mathrm{e}}$ & $(0.442-195.935)$ \\
\hline diabetes & lacuna & & & & \\
\hline$(-)$ & $(-)$ & 57 & 11 & 1.00 & (ref.) \\
\hline$(-)$ & $(+)$ & 38 & 6 & $0.684^{f}$ & $(0.212-2.205)$ \\
\hline
\end{tabular}




\begin{tabular}{cccccl}
$(+)$ & $(-)$ & 29 & 12 & $2.104^{\mathrm{f}}$ & $(0.784-5.648)$ \\
$(+)$ & $(+)$ & 31 & 43 & $7.179^{\mathrm{f}}$ & $\begin{array}{l}(2.996-17.202) \\
\end{array}$ \\
& & & Synergy index & $\mathbf{7 . 8 3 9}^{\mathrm{f}}$ & $(0.547-112.447)$ \\
\hline diabetes & lacuna & & & & \\
$(-)$ & $(-)$ & 57 & 11 & 1.00 & $($ ref.) \\
$(-)$ & $(+)$ & 38 & 6 & $0.629^{\mathrm{g}}$ & $(0.190-2.078)$ \\
$(+)$ & $(-)$ & 29 & 12 & $1.978^{\mathrm{g}}$ & $(0.724-5.405)$ \\
$(+)$ & $(+)$ & 31 & 43 & $7.084^{\mathrm{g}}$ & $(2.836-17.698)$ \\
& & & Synergy index & $\mathbf{1 0 . 0 1 8}^{\mathrm{g}}$ & $(0.344-291.414)$ \\
\hline
\end{tabular}

3 Abbreviations: CI, confidence interval; OR, odds ratio; ref, reference.

4 a without any adjusted.

5 b adjusted for age.

$6{ }^{c}$ adjusted for sex and age.

7 d adjusted for sex, age, and history of smoking.

8 e adjusted for age, sex, history of smoking, and education.

$9 \mathrm{f}$ adjusted for sex, age, smoking status, education, and hyperlipidemia.

$10 \mathrm{~g}^{\mathrm{g}}$ adjusted for sex, age, smoking status, education, hyperlipidemia, and hypertension. 